Published in final edited form as:

Infect Control Hosp Epidemiol. 2016 September ; 37(9): 1118-1120. doi:10.1017/ice.2016.154.

\title{
Reduction in Acute Respiratory Infection Among Military Trainees: Secondary Effects of a Hygiene-Based Cluster- Randomized Trial for Skin and Soft-Tissue Infection Prevention
}

\author{
Eugene V. Millar, PhD $^{1,2}$, Carey D. Schlett, MPH ${ }^{1,2}$, Natasha N. Law, MA ${ }^{1,2,3}$, Wei-Ju Chen, \\ PhD $^{1,2}$, Michael J. D’Onofrio, MD, MPH ${ }^{4}$, Jason W. Bennett, MD, MSPH ${ }^{5}$, David R. Tribble, \\ MD, DrPH ${ }^{1,5}$, and Michael W. Ellis, MD ${ }^{6}$
}

${ }^{1}$ Infectious Disease Clinical Research Program, Department of Preventive Medicine and Biostatistics, Uniformed Services University, Bethesda, Maryland ${ }^{2}$ Henry M. Jackson Foundation for the Advancement of Military Medicine, Rockville, Maryland ${ }^{3}$ Martin Army Community Hospital, Fort Benning, Georgia ${ }^{4}$ United States Army Medical Research Institute of Infectious Diseases, Medical Division, Frederick, Maryland ${ }^{5}$ Department of Medicine, Uniformed Services University, Bethesda, Maryland ${ }^{6}$ University of Toledo College of Medicine and Life Sciences, Toledo, Ohio

Military trainees are at increased risk for acute respiratory infection (ARI). ${ }^{1,2}$ ARI outbreaks interrupt training and compromise troop readiness. Mitigating the risk requires prevention strategies. A skin and soft-tissue infection (SSTI) prevention trial was conducted among Army trainees. ${ }^{3}$ Training companies were randomized to 1 of 3 groups with incrementally increasing education- and personal hygiene-based measures. The principal components were promotion of handwashing in addition to a once-weekly application of chlorhexidinebased body wash. Anticipating that these SSTI prevention measures would reduce the burden of other infections, ${ }^{4}$ we evaluated their impact on ARI. We observed a reduction in ARI among trainees who were educated on personal hygiene practices. The use of a chlorhexidine-based cleanser had no impact on ARI.

\section{METHODS}

We conducted a cluster-randomized SSTI prevention trial among Army trainees at Fort Benning, Georgia. ${ }^{3}$ There were 3 study groups (standard, enhanced standard, and chlorhexidine [CHG]), each with 10,000 trainees. Each group was assigned an intervention consisting of incrementally increasing education- and personal hygiene-based measures. ${ }^{3}$ The standard group trainees received a SSTI prevention brief upon entry. The enhanced standard group trainees received the standard group components in addition to supplemental

\footnotetext{
Address correspondence to Eugene V. Millar, PhD, Infectious Disease Clinical Research Program, Department of Preventive Medicine and Biostatistics, Uniformed Services University of the Health Sciences, 11300 Rockville Pike, Suite 1211, Rockville, MD 20852 (emillar@idcrp.org); Carey D. Schlett, MPH, Infectious Disease Clinical Research Program, Department of Preventive Medicine and Biostatistics, Uniformed Services University of the Health Sciences, 11300 Rockville Pike, Suite 1211, Rockville, MD 20852 (cschlett@idcrp.org).

Potential conflicts of interest: All authors report no conflicts of interest relevant to this article.

CLINICAL TRIALS REGISTRATION. MRSA SSTI Prevention in Military Trainees (ClinicalTrials.gov Identifier: NCT01105767; URL: clinicaltrials.gov/show/NCT01105767).
} 
materials (ie, a pocket card and posters in the barracks). The CHG group trainees received the enhanced standard group components in addition to a CHG-based body wash (Hibiclens, Mölnlycke Heath Care, Norcross, Georgia). Trainees were instructed to use the wash once weekly for the entire training period. All trainees sought care at a single outpatient clinic.

For a planned secondary objective of the trial, we reviewed an electronic database (Armed Forces Health Longitudinal Technology Application, AHLTA) to identify medically attended, outpatient cases of ARI in the study population. The case definition was any occurrence of the following International Classification of Disease, $9^{\text {th }}$ Revision, Clinical Modification (ICD-9) symptom or disease-specific codes: 460-466, 480-488, and specifically 465.9, 482.9, 486, and 487.1. Data abstractors were blinded to group assignment.

Rate calculations included all ARI-associated visits, allowing multiple visits per individual. Rates are the number of cases per 1,000 person weeks. Binomial distributions were used to generate 95\% confidence intervals (CIs). Rate ratios (RRs) were compared using Fisher's exact test.

Statistical analyses were performed using Microsoft Excel (Microsoft Corporation, Redmond, WA), Stata 12.1 (StataCorp, College Station, TX), and SAS 9.3 (SAS Institute, Cary, NC).

\section{RESULTS}

Over a 20-month period and among 30,000 trainees, a total of 13,949 ARI episodes were identified: 4,365 (31.3\%) in the standard group; 4,426 (31.7\%) in the enhanced standard group; and 5,158 (36.9\%) in the CHG group (Table 1). The overall ARI rate was 33.9 cases per 1,000 person weeks (95\% CI, 33.3-34.5). By study group, ARI rates were 35.3 per 1,000 person weeks in the standard group (95\% CI, 34.3-36.3); 29.3 in the enhanced standard group (95\% CI, 28.5-30.2); and 37.7 in the CHG group (95\% CI, 36.7-38.7). When compared with the standard group, ARI rates were lower in the enhanced standard group (RR, 0.82; 95\% CI, 0.80-0.87) and marginally higher in the CHG group (RR, 1.07; 95\% CI, 1.03-1.11). The enhanced standard:CHG group RR was 0.78 (95\% CI, 0.75-0.81).

Overall rates were highest in winter. By season, enhanced standard:standard RRs were as follows: summer (RR, 0.77; 95\% CI, 0.72-0.83), fall (RR, 0.97; 95\% CI, 0.91-1.05), winter (RR, 0.93; 95\% CI, 0.84-1.03), and spring (RR, 0.63; 95\% CI, 0.57-0.70). When compared to the standard group, ARI rates in the CHG group were lower only in the spring (RR, 0.79; 95\% CI, 0.72-0.86).

Case characteristics are presented in the Table 1 . The most common code was acute upper respiratory infection not otherwise specified $(\mathrm{n}=7,182 ; 52.9 \%)$. Other frequent diagnoses were acute pharyngitis $(\mathrm{n}=1,749 ; 12.9 \%)$, acute nasopharyngitis $(\mathrm{n}=1,694 ; 12.5 \%)$, and pneumonia, organism unspecified $(n=1,458 ; 10.9 \%)$. The distribution of diagnoses did not differ by study group. 


\section{DISCUSSION}

In the context of an SSTI prevention trial, we observed a reduction in ARI rates among military trainees who received educational briefs and materials on personal hygiene practices. When compared with the standard group, ARI rates in the enhanced standard group were $\sim 20 \%$ lower, strengthening the evidence that promotion of good hand hygiene can reduce the burden of ARI in the military training setting, where crowded living conditions and frequent physical contact puts trainees at increased risk for disease. Use of a chlorhexidine-based cleanser had no impact on ARI.

The findings of our study are consistent with other military-based ARI prevention interventions. Promotion of hand hygiene (ie, frequent handwashing, use of hand sanitizer) among military recruits was associated with $40 \%-45 \%$ reductions in respiratory illness. 5,6 While the primary focus of our trial was SSTI prevention, it is likely that overall improvement in personal hygiene practices, namely handwashing, interrupted the transmission of respiratory pathogens and prevented secondary ARI cases in this vulnerable population. ${ }^{7}$

This investigation had both strengths and weaknesses. This study was nested in a large fieldbased, randomized controlled trial and utilized clinic-based medical records. Because trainees sought care at a single medical clinic, we captured all clinical encounters. However, we did not capture cases for those who either did not seek care for their illness, or who presented directly to the hospital, nor did we have information on etiology. Lastly, we did not measure the trainees' frequency of personal hygiene practices during the training period.

In summary, we observed a reduction in ARI rates among military trainees who received additional education on personal hygiene practices. These same measures were associated with a reduction in acute gastroenteritis in this population. ${ }^{4}$ We conclude that routine promotion of personal hygiene, especially hand hygiene, remains an important strategy for the prevention of infectious diseases among military trainees.

\section{Acknowledgments}

\footnotetext{
We are indebted to the study team of clinical research coordinators, laboratory personnel, and data management staff for their dedication to the project. The views expressed in this paper are those of the authors and do not necessarily represent the views of the Uniformed Services University of the Health Sciences, the Department of Defense (DoD), or the Henry M. Jackson Foundation for the Advancement of Military Medicine.
}

Financial support: The work was supported by the Infectious Disease Clinical Research Program (IDCRP), a Department of Defense (DoD) program executed through the Uniformed Services University of the Health Sciences. This project was funded in part with federal funds from the National Institute of Allergy and Infectious Diseases, National Institutes of Health (NIH), under Inter-Agency Agreement (grant no. Y1-AI-5072). Additional funding was provided by Centers for Disease Control and Prevention, National Center for Emerging and Zoonotic Infectious Diseases, Division of Healthcare Quality Promotion (NCEZID-DHQP); Interagency Agreement (grant no. 09FED914272 to MWE).

\section{References}

1. O’Shea MK, Ryan MA, Hawksworth AW, Alsip BJ, Gray GC. Symptomatic respiratory syncytial virus infection in previously healthy young adults living in a crowded military environment. Clin Infect Dis. 2005; 41:311-317. [PubMed: 16007526] 
2. Russell KL, Hawksworth AW, Ryan MA, et al. Vaccine-preventable adenoviral respiratory illness in US military recruits, 1999-2004. Vaccine. 2006; 24:2835-2842. [PubMed: 16480793]

3. Ellis MW, Schlett CD, Millar EV, Wilkins KJ, Crawford KB, Morrison-Rodriguez SM, et al. Hygiene strategies to prevent methicillin-resistant Staphylococcus aureus skin and soft tissue infections: a cluster-randomized controlled trial among high-risk military trainees. Clin Infect Dis. 2014; 58:1540-1548. [PubMed: 24633684]

4. D'Onofrio MJ, Schlett CD, Millar EV, et al. Reduction in acute gastroenteritis among military trainees: secondary effects of a hygiene-based cluster-randomized trial for skin and soft tissue infection prevention. Infect Control Hosp Epidemiol. 2015; 36:358-360. [PubMed: 25695181]

5. Ryan MA, Christian RS, Wohlrabe J. Handwashing and respiratory illness among young adults in military training. Am J Prev Med. 2001; 21:79-83. [PubMed: 11457626]

6. Mott PJ, Sisk BW, Arbogast JW, Ferrazzano-Yaussy C, Bondi CA, Sheehan JJ. Alcohol-based instant hand sanitizer use in military settings: a prospective cohort study of Army basic trainees. Mil Med. 2007; 172:1170-1176. [PubMed: 18062391]

7. Jefferson T, Del Mar CB, Dooley L, et al. Physical interventions to interrupt or reduce the spread of respiratory viruses. Cochrane Database Syst Rev. 2011:CD006207, 1-162. [PubMed: 21735402] 


\section{TABLE 1}

Incidence Density and Case Characteristics of Acute Respiratory Infection Among US Army Trainees Participating in a Cluster-Randomized Trial Utilizing Personal Hygiene-Based Measures

\begin{tabular}{|c|c|c|c|}
\hline & \multicolumn{3}{|c|}{ Study Group } \\
\hline & Standard & $\begin{array}{l}\text { Enhanced Standard, No. } \\
\qquad(95 \% \mathrm{CI})\end{array}$ & $\begin{array}{l}\text { Chlorhexidine, No. } \\
\qquad(95 \% \text { CI })\end{array}$ \\
\hline No. of episodes & 4,365 & 4,426 & 5,158 \\
\hline Incidence density $(95 \% \mathrm{CI})^{a}$ & $35.3(34.3-36.3)$ & $29.3(28.5-30.2)$ & $37.7(36.7-38.7)$ \\
\hline Rate ratio $(95 \% \mathrm{CI})^{b}$ & $\cdots$ & $0.82(0.80-0.87)$ & $1.07(1.03-1.11)$ \\
\hline No. of cases & 2,803 & 2,941 & 3,353 \\
\hline $\begin{array}{l}\text { Median (range) no. of days from training start to first ARI } \\
\text { episode }\end{array}$ & $26(2-98)$ & $36(6-98)$ & $31(3-89)$ \\
\hline No. $(\%)$ of subjects with $>1$ visit for ARI & $975(34.8)$ & $954(32.4)$ & $1,177(35.1)$ \\
\hline
\end{tabular}

NOTE. CI, confidence interval.

${ }^{a}$ Reported as the number of cases per 1,000 person weeks.

$b_{\text {Standard group as reference. }}$ 